CLINICAL RESEARCH ARTICLE

\title{
Autonomic development in preterm infants is associated with morbidity of prematurity
}

Sarah D. Schlatterer ${ }^{1,2,3}$, Rathinaswamy B. Govindan ${ }^{1,3}$, Scott D. Barnett ${ }^{1}$, Tareq Al-Shargabi ${ }^{1}$, Daniel A. Reich ${ }^{1}$, Sneha lyer ${ }^{4}$, Laura Hitchings ${ }^{1}$, G. Larry Maxwell ${ }^{5}$, Robin Baker ${ }^{5,6}$, Adre J. du Plessis ${ }^{1,2,3}$ and Sarah B. Mulkey ${ }^{1,2,3}$

BACKGROUND: Previous studies have described an association between preterm birth and maturation of the autonomic nervous system (ANS); however, this may be impacted by multiple factors, including prematurity-related complications. Our aim was to evaluate for the effect of prematurity-related morbidity on ANS development in preterm infants in the NICU.

METHODS: We compared time and frequency domains of heart rate variability (HRV) as a measure of ANS tone in 56 preterm infants from 2 NICUs (28 from each). One cohort was from a high-morbidity regional referral NICU, the other from a communitybased inborn NICU with low prematurity-related morbidity. Propensity score matching was used to balance the groups by a 1:1 nearest neighbor design. ANS tone was analyzed.

RESULTS: The two cohorts showed parallel maturational trajectory of the alpha 1 time-domain metric, with the cohort from the high-morbidity NICU having lower autonomic tone. The maturational trajectories between the two cohorts differed in all other time-domain metrics (alpha 2, RMS1, RMS2). There was no difference between groups by frequency-domain metrics.

CONCLUSIONS: Prematurity-associated morbidities correlate with autonomic development in premature infants and may have a greater impact on the extrauterine maturation of this system than birth gestational age.

Pediatric Research (2022) 91:171-177; https://doi.org/10.1038/s41390-021-01420-x

\section{IMPACT:}

- Autonomic nervous system development measured by time-domain metrics of heart rate variability correlate with morbidities associated with premature birth.

- This study builds upon our previously published work that showed that development of autonomic tone was not impacted by gestational age at birth.

- This study adds to our understanding of autonomic nervous system development in a preterm extrauterine environment.

- Our study suggests that gestational age at birth may have less impact on autonomic nervous system development than previously thought.

\section{INTRODUCTION}

An appropriately mature autonomic nervous system (ANS) is key for successful transition from the intrauterine to extrauterine environment at birth. Abnormal ANS development has been linked to neurodevelopmental, neuropsychiatric, and cardiovascular outcomes later in life. ${ }^{1-4}$ The ANS undergoes a critical period of maturation during the latter half of gestation and during the early neonatal period. Previous studies have described an association between preterm birth and subsequent extrauterine maturation on the developing ANS. $^{5-8}$ However, there are multiple potential factors beyond birth gestational age (GA) that may influence ANS development in the extrauterine environment. We recently studied a relatively low-morbidity population of premature infants and found no significant association between duration of preterm extrauterine maturation and metrics of ANS development from birth to term-corrected age. ${ }^{9}$ We proposed that birth GA may have less impact on ANS development than previously reported.

Prior studies investigating ANS maturation in preterm infants have usually involved infants with higher levels of prematurityrelated complications. ${ }^{6-8}$ Consequently, ANS development in preterm infants may be related more to medical comorbidities than to birth GA itself. To address this question, we sought to compare ANS development in preterm infants from two neonatal intensive care units (NICUs) with different levels of prematurityrelated morbidities. In this study, we set out to determine the degree to which complications of prematurity impact ANS development by comparing two populations of preterm infants with differing levels of complications of prematurity.

\footnotetext{
${ }^{1}$ Children's National Hospital, Prenatal Pediatrics Institute, Washington, DC, USA; ${ }^{2}$ Department of Neurology, The George Washington University School of Medicine and Health Sciences, Washington, DC, USA; ${ }^{3}$ Department of Pediatrics, The George Washington University School of Medicine and Health Sciences, Washington, DC, USA; ${ }^{4}$ The George

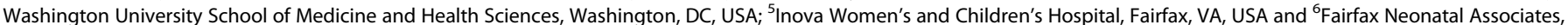
Fairfax, VA, USA

Correspondence: Sarah D. Schlatterer (sschlatt@childrensnational.org)
}

Received: 28 October 2020 Revised: 5 January 2021 Accepted: 2 February 2021

Published online: 2 March 2021 
172

\section{METHODS}

Participants

We compared a panel of time- and frequency-domain ANS metrics in preterm infants (birth $\mathrm{GA} \leq 34$ weeks) from two different level-4 NICUs. The lower morbidity (LOW) NICU at Inova Fairfax Hospital is a large (108 bed) suburban community unit caring for inborn infants from a large obstetric program with about 10,000 deliveries per year. In a longitudinal prospective study of ANS maturation between May 2017 and May 2019, preterm infants were enrolled within $96 \mathrm{~h}$ of birth. Infants with a birth GA of $231 / 7$ to $336 / 7$ weeks, requiring NICU admission, without a suspected genetic condition at the time of enrollment, and expected to survive to NICU discharge at the time of enrollment were included in the study. The higher morbidity cohort $(H I G H)$ cohort was recruited from the Children's National Hospital NICU, an urban quaternary referral center, between June 2017 to November 2018, and included preterm infants with the same eligibility criteria. These subjects were retrospectively enrolled and their medical records and archived continuous physiological signals were analyzed. All infants in the Children's National cohort $(H I G H)$ were outborn and admitted before $72 \mathrm{~h}$ of age for medical or surgical complications of prematurity.

For the purposes of this study, we recorded significant postnatal medical complications at any time during the subjects' NICU admission, including any of the following: culture-positive infection occurring at $<7$ days and $\geq 7$ days of life (infection), necrotizing enterocolitis (NEC) diagnosis requiring medical or surgical intervention, presence of a patent ductus arteriosus (PDA) requiring medical or surgical intervention, brain injury on head ultrasound including periventricular leukomalacia and intraventricular hemorrhage grade 3 or 4 (neuro), diagnosis of bronchopulmonary dysplasia or chronic lung disease, retinopathy of prematurity at stage $\geq 1$, and need for mechanical ventilation/ intubation (mechanical ventilation). We also added up the total number of above conditions for each subject in the study for a summative "comorbidity score." Data on medical comorbidities was abstracted from the medical record. We added the total number of the above conditions for each subject in the study to calculate a "comorbidity score." The studies were approved by the Children's National Hospital and INOVA Hospital Institutional Review Boards.

\section{Physiologic signal collection}

In the INOVA cohort (LOW), we collected up to $96 \mathrm{~h}$ of continuous electrocardiogram (ECG) recording directly from the infants' bedside monitor immediately following enrollment and arrival in the NICU using custom software developed in either MATLAB 2018 (Mathworks, Inc., Natick, MA) or Labview (National Instruments, Austin, TX). The sampling rate of the ECG was $1000 \mathrm{~Hz}$. After this initial prolonged ECG data collection period, we recorded up to $7 \mathrm{~h}$ of ECG data during the daytime at weekly intervals until NICU discharge.

In the Children's National cohort $(H I G H)$, ECG data were retrospectively retrieved from the Phillips Data Warehouse system (DWS) (IntelliVue MP-70, Philips, MA). We retrieved ECG data at weekly intervals from the Children's National NICU admission until 42 weeks corrected GA during daytime hours, with the first collection occurring within the first $72 \mathrm{~h}$ of life in order to match the timing of the initial and weekly ECG data collection periods in the INOVA (LOW) cohort. The sampling rate of the ECG retrieved from the DWS is $250 \mathrm{~Hz}$. All data were processed offline using computer programs developed in MATLAB.

Heart rate variability (HRV) analysis

We bandpass filtered the ECG signals between 0.5 and $60 \mathrm{~Hz}$, identified R-waves, and calculated beat-to-beat interval (RRi). ${ }^{10,11}$ We partitioned RRi into 10-min end-to-end epochs and calculated HRV metrics for RRi in every epoch. We estimated a power spectrum of RRi in every 10-min epoch using the Welch periodogram approach with a frequency resolution of $0.0167 \mathrm{~Hz}$. We calculated low frequency (LF) and high frequency (HF) as the median of the logarithm of the power in $0.05-0.25$ and $0.3-1 \mathrm{~Hz}$, respectively. We also calculated normalized low frequency (nLF) and normalized high frequency (nHF) as the ratio of the sum of the powers in low- and high-frequency bands to the total power. Total power was defined as the sum of the powers in the frequency band between 0.05 and $2 \mathrm{~Hz}$.

To calculate the time-domain metrics, we performed the detrended fluctuation analysis (DFA), which is a modified root mean square (RMS) analysis. In short, DFA involves the following five steps. First, the mean value of the RRi is subtracted from the RRi series and the cumulative sum, called the profile function, is calculated. Second, the profile function is partitioned into end-toend windows containing " $s$ " number of beats and fit to the profile in each window using a fourth-degree polynomial. Third, the local fluctuation function is calculated as the RMS of the difference between the profile and best fits. Fourth, the fluctuation function is calculated as the median of the local fluctuation function from all the windows. Last, steps 1-3 are repeated for different window sizes. ${ }^{12}$ In our calculation, we varied the window size from 6 beats to one-fourth of the number of RRi samples in the 10-min epoch. Using the DFA fluctuation function, we calculated time-domain metrics as follows. ${ }^{13,14}$ RMS1 (s) and RMS2 (s) were calculated as the maximum value of the fluctuation function for " $s$ " between 15 and 50 beats and 100-150 beats, respectively. We also calculated alpha 1 and alpha 2 as the slope of the fluctuation and " $s$ " in double logarithmic plot from the regions of 15-30 beats (shortterm scale) and 35-150 beats (long term scale/ultralow frequency), respectively. The alpha metrics characterize the autocorrelation in the RRi, while the RMS (s) metrics characterize the variability in the RRi. ${ }^{15}$ We calculated eight HRV metrics (LF, HF, nLF, nHF, alpha 1, alpha 2, RMS1, RMS2) in every 10-min epoch and averaged every metric over the entire recording period and obtained one representative value for the metric per study. In our earlier study, we showed that HF power calculated from DWS is compromised and hence we excluded this metric from further analysis.

For infants on the invasive ventilator support, RRi contains respiration-induced (artificial) oscillations in the short time scale. These oscillations affect the estimation of the alpha 1 estimation. To attenuate the artificial respiratory oscillations, we reconstructed the respiratory waves using the amplitude changes in the ECG signal. Using the reconstructed wave, we attenuated the respiratory components in the RRi using a frequency-dependent subtraction approach. We calculated alpha 1 using the respiration attenuated RRi.

Propensity score matching (PSM)

To balance the HIGH and LOW morbidity cohorts, PSM was used. The probability that a subject was in the HIGH cohort given a set of clinically important variables such as sex, maternal age, birth GA, birth weight Z-score, birth head circumference Z-score, maximum APGAR score, and length of stay was estimated using an unconditional multivariate logistic regression model. We then implemented one-to-one (1:1) nearest neighbor matching design.

Statistical analysis

Following matching, we performed separate latent growth modeling using a repeated-measures random-effects mixed model approach to determine the relationship between postnatal age (PNA) days on the selected variables. PNA days were treated as random effects. Models were assessed for a second-order polynomial function, and for consistency across models, given that only one term (alpha 1) was quadratic, a decision was made to treat PNA days as a linear function. Several various covariance matrices were evaluated using Akaike's Information Criterion with an unstructured covariance matrix determined to be best fitting. 
Table 1. Demographic data.

\begin{tabular}{|c|c|c|c|}
\hline Demographic data & $H I G H(n=28)$ & $\operatorname{LOW}(n=28)$ & $p$ value \\
\hline Gestational age at birth (weeks) & 28.8 (27.3 to 30.4$)$ & 28.6 (26.2 to 30.2 ) & 0.58 \\
\hline Z-score & $-0.10(-0.69$ to 0.55$)$ & $0.05(-0.41$ to 0.39$)$ & \\
\hline Head circumference $(\mathrm{cm})$ & 26.0 (23.3 to 28.0$)$ & 26.5 (23.5 to 29.0$)$ & 1.0 \\
\hline Apgar $5 \mathrm{~min}$ & 7.5 (6.0 to 9.0$)$ & 8.0 (7.0 to 9.0$)$ & \\
\hline \multicolumn{4}{|l|}{ Sex } \\
\hline Female & $15(54)$ & $11(39)$ & 0.28 \\
\hline Male & $13(46)$ & $17(61)$ & \\
\hline \multicolumn{4}{|l|}{ Delivery type } \\
\hline
\end{tabular}

Two-sided significance testing was used. All analyses were conducted using SAS (ver. 9.3, Cary, NC) with statistical significance considered for $p<0.05$. No adjustment was made for multiple comparisons. Descriptive and comparative statistics were performed for patient baseline characteristics and treatment outcomes. Data are presented as median (Q1-Q3) or frequency and percentage. Group comparisons were made using either $t$ tests or Fisher's exact test, where appropriate.

\section{RESULTS}

We evaluated autonomic metrics over time from birth to NICU discharge or 42 weeks corrected GA in 56 infants (28 in each of the $H I G H$ and LOW cohorts), propensity score-matched to minimize the effects of treatment site. The clinical and demographic characteristics of the infants are reported in Table 1. There was no significant difference in demographic characteristics, as expected, due to propensity matching. LOW infants were more likely to have been delivered by cesarean section $(p=0.04)$. Prenatal and postnatal conditions of the pregnant women and infants are presented in Table 2. There was no significant difference in the rates of multiple gestation, fetal growth restriction/intrauterine growth restriction, pre-eclampsia, or pregnancy-induced hypertension between the cohorts. However, LOW infants were more likely to have received antenatal steroids than HIGH infants $(p=0.03)$. HIGH infants had higher rates of PDA $(p=0.002)$, culture-positive infections $(p=$ $0.053)$, and mechanical ventilation $(p=0.002)$ and had higher number of comorbidities per infant than LOW infants (average no. of comorbidities $2.6 \pm 1.8$ vs. $1.3 \pm 1.4, p=0.003$ ).

Both $H I G H$ and LOW cohorts showed changes over time for all autonomic metrics (Figs. 1 and 2 and Table 3). There was an absolute difference in the alpha 1 measure between $H I G H$ and LOW cohorts, shown by nearly parallel maturational trajectories in Fig. 1 and in a significant cohort effect in Table 3. No other HRV metric showed a similar difference between cohorts. All other time-domain metrics (alpha 2, RMS1, RMS2) showed significant differences in the group-by-time interaction term (Table 3), reflecting different maturational trajectories between the groups. RMS1 and RMS2 absolute values were similar between cohorts soon after delivery, but the curves for the two groups diverged, with the LOW cohort showing more robust development than the $H I G H$ cohort over time (Fig. 1). Alpha 2 was lower in the HIGH
Table 2. Prenatal and postnatal comorbidities.

\begin{tabular}{|c|c|c|c|}
\hline & $H I G H(n=28)$ & $\operatorname{LOW}(n=28)$ & $p$ value \\
\hline \multicolumn{4}{|l|}{ Prenatal } \\
\hline Multiple gestation & $5(18)$ & $2(7)$ & 0.42 \\
\hline FGR/IUGR & $5(18)$ & $2(7)$ & 0.42 \\
\hline Pre-eclampsia & $5(18)$ & $8(29)$ & 0.53 \\
\hline $\begin{array}{l}\text { Pregnancy- } \\
\text { induced HTN }\end{array}$ & $2(7)$ & $0(0.0)$ & 0.75 \\
\hline Steroids & $17(61)$ & $25(89)$ & 0.03 \\
\hline \multicolumn{4}{|l|}{ Postnatal } \\
\hline Length of stay & $70.5(56.0-90.0)$ & $74.6(42.0-106.6)$ & 0.56 \\
\hline Neuro & $7(25)$ & $2(7)$ & 0.14 \\
\hline NEC & $2(7)$ & $1(4)$ & 1.0 \\
\hline ROP stage $\geq 1$ & $9(32)$ & $7(25)$ & 0.77 \\
\hline $\mathrm{BPD} / \mathrm{CLD}$ & $14(50)$ & $13(46)$ & 1.0 \\
\hline PDA & $11(39)$ & $1(7)$ & 0.002 \\
\hline ID & $6(21)$ & $0(0.0)$ & 0.053 \\
\hline$<7$ days of life & $1(17)$ & $0(0.0)$ & - \\
\hline$\geq 7$ days of life & $5(83)$ & $0(0.0)$ & 0.43 \\
\hline Mechanical ventilation & $23(82)$ & $11(39)$ & 0.002 \\
\hline $\begin{array}{l}\text { Average no. of } \\
\text { comorbidities }\end{array}$ & $2.6 \pm 1.8$ & $1.3 \pm 1.4$ & 0.003 \\
\hline \multicolumn{4}{|c|}{$\begin{array}{l}\text { Categorical data represented as } N(\%) \text { and continuous data are reported as } \\
\text { median (Q1-Q3) or as mean (SD); } p \text { values are calculated by Fisher's } \\
\text { Exact Test. } \\
\text { Bold values indicate statistical significance } p<0.05 \text {. }\end{array}$} \\
\hline
\end{tabular}

cohort compared with the LOW cohort at early time points but was higher in the HIGH cohort close to term equivalent age (Fig. 1). There was no difference between the frequency-domain metrics by cohort (LF, nLF, nHF; Table 3 and Fig. 2).

\section{DISCUSSION}

In this study, we found that ANS developmental trajectory in preterm-born infants may relate more to prematurity-associated 

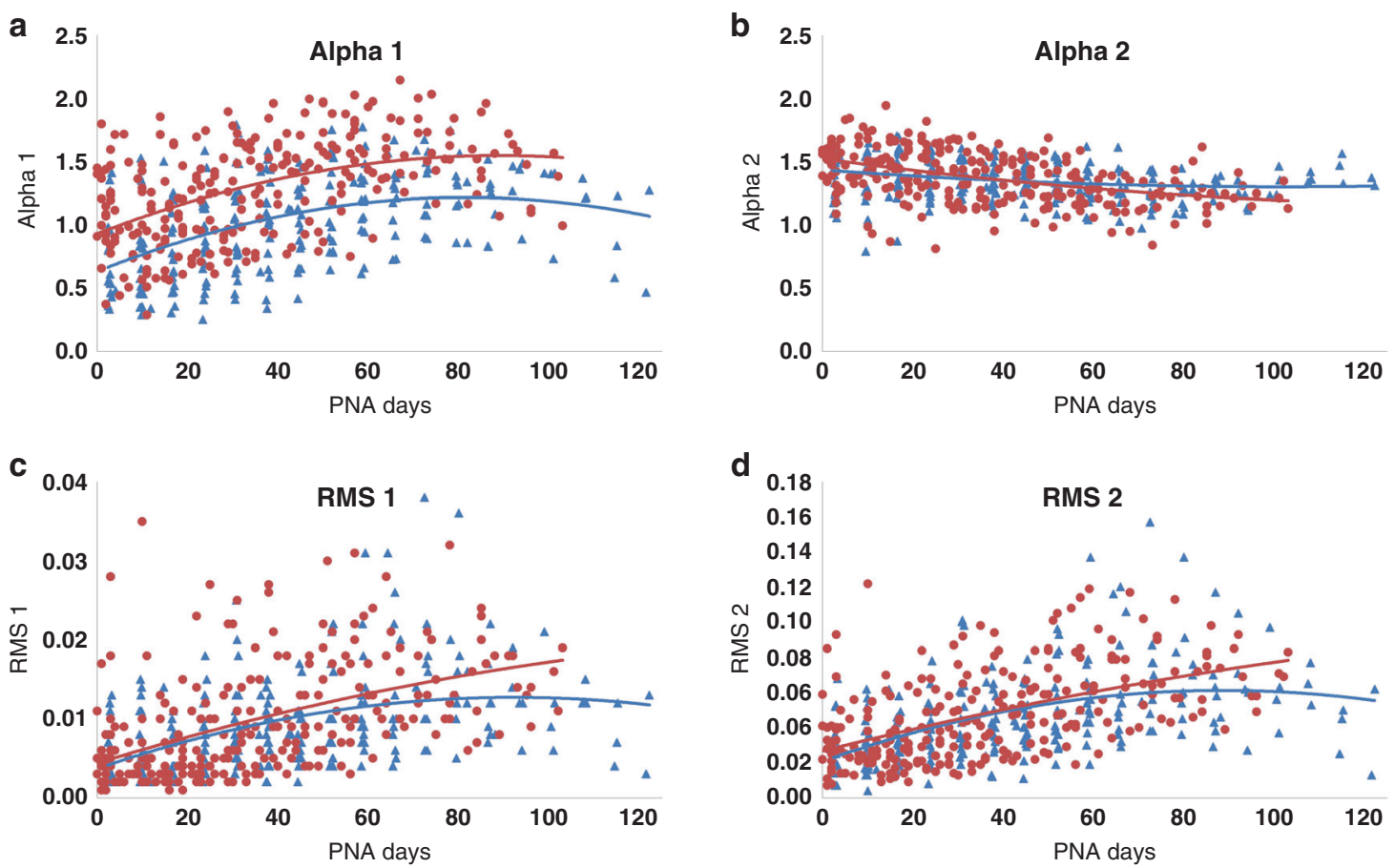

Fig. 1 Trajectory of development of time-domain metrics of heart rate variability. Children's cohort (HIGH) in blue, INOVA cohort (LOW) in red. PNA = postnatal age in days. a Alpha 1 development. b Alpha 2 development. c RMS1 development. d RMS2 development.
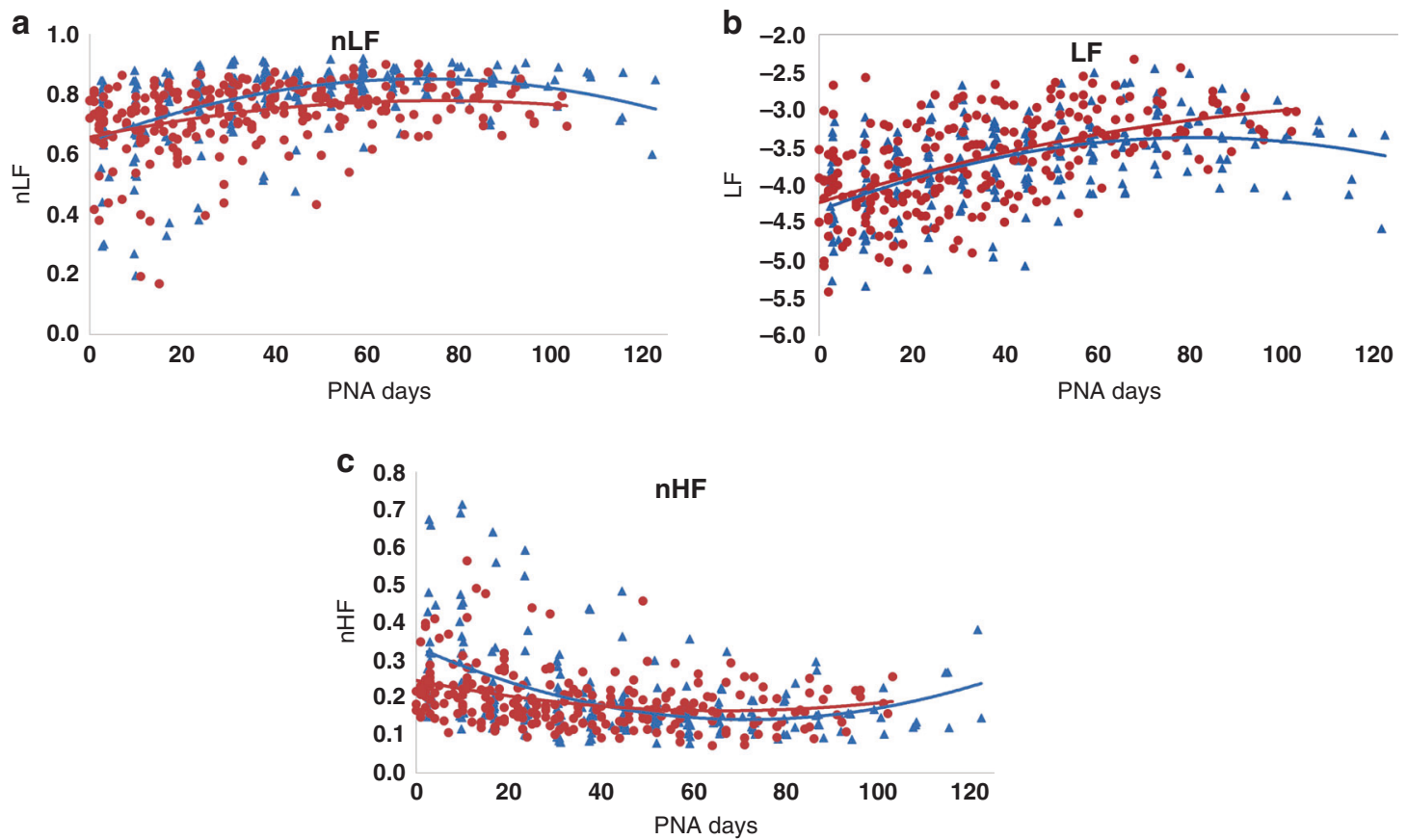

Fig. 2 Trajectory of development of frequency-domain metrics of heart rate variability. Children's National cohort (HIGH) in blue, INOVA cohort (LOW) in red. a Normalized low frequency (nLF) development. b Low frequency (LF) power development. c Normalized high frequency (nHF) development.

medical comorbidity than to birth GA itself. In a preterm infant cohort with a low level of medical morbidity, we recently demonstrated that duration of extrauterine development did not seem to significantly impact ANS developmental trajectory from birth to NICU discharge. ${ }^{9}$ Our finding is in contrast to previous other studies in which significant effects of prematurity on ANS development were reported. ${ }^{7-9}$ These previous reports included preterm populations with higher levels of medical complexity. ${ }^{7-9}$ The data from our current study supports the hypothesis that the medical complexity, in addition to birth GA, has an impact on ANS development in preterm infants.

The ANS undergoes a prolonged period of development that spans the fetal period and continues after birth. ${ }^{16,17}$ The sympathetic division of the ANS begins to develop early in gestation shortly after the unmyelinated vagus (which has little function until after birth despite early structural development ${ }^{18}$ ) and continues to steadily 
Table 3. Time, cohort, and cohort by time interaction effects.

\begin{tabular}{|c|c|c|c|c|c|c|c|c|c|c|c|c|c|c|}
\hline & \multicolumn{2}{|c|}{ Alpha 1} & \multicolumn{2}{|c|}{ Alpha 2} & \multicolumn{2}{|l|}{ RMS1 } & \multicolumn{2}{|l|}{ RMS2 } & \multicolumn{2}{|l|}{$\mathrm{nHF}$} & \multicolumn{2}{|l|}{$\mathrm{nLF}$} & \multicolumn{2}{|l|}{ LF } \\
\hline Time effect & 76.0 & $<0.001$ & 122.6 & $<0.001$ & 139.3 & $<0.001$ & 165.2 & $<0.001$ & 32.8 & $<0.001$ & 37.6 & $<0.001$ & 154.4 & $<0.001$ \\
\hline Cohort effect & 7.4 & 0.03 & 3.4 & 0.226 & 0.4 & 0.56 & 0.1 & 0.75 & 2.2 & 0.14 & 0.7 & 0.41 & 0.03 & 0.85 \\
\hline Cohort $\times$ time interaction & 0.6 & 0.05 & 29.4 & $<0.001$ & 14.0 & $<0.001$ & 6.9 & 0.01 & 2.8 & 0.09 & 1.0 & 0.32 & 2.5 & 0.11 \\
\hline
\end{tabular}

mature throughout the fetal period. In the third trimester, the myelinated vagus nerve develops and begins to exert parasympathetic influence, ${ }^{18,19}$ resulting in a steep increase in parasympathetic tone near term.,20,21 Within the central ANS, brainstem and hypothalamic centers develop first and develop multiple, complex connections with supratentorial structures, including the insula, the anterior thalamus, the anterior cingulate gyrus, the hippocampus, the hypothalamus, and the amygdala, later in gestation and during the early neonatal period. ${ }^{5,22}$ Overall, sympathetic innervation develops and begins exerting influence earliest, with an increase in parasympathetic influence over time as higher cortical processes begin to integrate autonomic control during late gestation and the early neonatal period. ${ }^{23,24}$

HRV, the variation in beat-to-beat (R-R) intervals between heart beats, is the most widely used measure of autonomic function in the neonate. HRV metrics can be analyzed by both time and frequency-domain approaches. Short-term variability in timedomain metrics reflects parasympathetic function, while longterm variability is influenced by both sympathetic and parasympathetic function. ${ }^{25}$ Sympathetic and parasympathetic tone and changes in heart rate related to baroreflex function are characterized by LF HRV, ${ }^{26,27}$ while HF HRV reflects parasympathetic tone. ${ }^{27}$

In our study, there were significant differences in rates of culture-positive infection, PDA requiring treatment, need for mechanical ventilation, and overall number of comorbidities between the two cohorts. Infectious and inflammatory conditions such as neonatal sepsis and NEC are known to be associated with short-term depression and alterations of autonomic tone, which may precede symptoms of infection and inflammation by hours to days. ${ }^{28-33}$ Preterm infants with early-onset sepsis are more likely to have lower ANS tone and abnormal neurologic outcomes. ${ }^{4}$ Autonomic tone has previously been shown to differ in infants with specific types of medical comorbidities, including structural heart disease, ${ }^{34}$ PDA, ${ }^{35}$ and hypoxic-ischemic encephalopathy (HIE) ${ }^{28,36}$ In term infants with HIE and stroke, brain injury has been associated with alterations in ANS function in the neonatal period. ${ }^{37,38}$ This study adds to the current body of knowledge by showing that more medically complex preterm infants as a group have ANS maturation different from that of preterm infants with fewer medical comorbidities.

Alpha 1 is a variable that reflects quality of regulation of the ANS and is the only HRV metric studied for which there was an absolute difference between cohorts, with nearly parallel trajectories of development. In this study, alpha 1 started and remained lower in the HIGH cohort compared with the LOW cohort, suggesting lower autonomic regulation. Whether this difference is due to the underlying medical complexity within the Children's National cohort $(H I G H)$ or is an inherent preceding factor that increases risk for medical complications in preterm infants that would necessitate transfer to a quaternary referral NICU cannot be determined from this study. This is a question worthy of further investigation. The role of prenatal factors should also be examined. Our LOW and HIGH cohorts differed only in the administration of antenatal steroids, and we cannot say from this study whether this had any influence on ANS development or on alpha 1. Studies focused on prenatal influence on ANS function and fetal HRV may enable determination of whether infant differences precede birth and the development of early postnatal medical complications.

Alpha 2 decreased over time in the LOW cohort (as we have shown previously ${ }^{9}$ ) but increased over time in the HIGH cohort. This finding may reflect sympathetic over-compensation, perhaps due to greater prevalence of painful or stressful events in the HIGH cohort. RMS1 and RMS2 metrics were very similar between cohorts early after birth, but trajectories diverged over time, suggesting that maturation is slowed in the HIGH cohort. Given that our two cohorts are matched for major demographic criteria but differ in their levels of medical complexity, these data suggest that levels of medical complexity have a significant impact on ANS development as measured by time-domain metrics. However, given the lack of difference in frequency-domain metrics, there are likely multiple influences on ANS development in the preterm infant with medical complexity being one factor.

While our study focused on postnatal comorbidities, we would be remiss not to mention potential prenatal differences between the cohorts that could have contributed to alterations in ANS development. To this end, we found no difference between prenatal factors studied, except the rate of antenatal steroids. We hypothesize that pregnant mothers in the LOW cohort, who are more likely to be of higher socioeconomic status, may have utilized prenatal care to a greater extent than the HIGH cohort, resulting in earlier diagnosis of risk for preterm labor and greater rates of administration of steroids. However, due to the retrospective nature of this study, we cannot determine with certainty why this difference was present. We found similar time-domain metrics between groups at similar PNAs that diverged over time in the NICU. While prenatal factors are critical for early ANS development, the altered trajectory of postnatally measured RMS1 and RMS2 metrics of ANS development occurred over time in the NICU and differed between the cohorts. Interestingly, alpha 1 differed between cohorts at the time of birth. Future studies are needed to separate the influence of prenatal vs. postnatal factors on different metrics of HRV and their contribution to ANS developmental trajectory.

Our study has a number of strengths, including the longitudinal HRV recordings in two preterm infant cohorts from different NICUs, but also has limitations. First, we used preterm populations with different levels of medical comorbidity from two separate NICU sites. This was necessary in order to have an appropriate number of cases in each group for comparison, and we attempted to minimize the impact of the possible differences in care/hospital population through propensity matching. Nevertheless, there may be differences between NICU care and populations that we are not able to address in this study analysis. We chose to report the data as PNA rather than postmenstrual age in this study, in order to show ANS maturation for infants who had been matched for GA at birth. The median GA at birth in our two cohorts was slightly higher than in previous studies. ${ }^{8}$ We do not have follow-up HRV measurements in these cohorts following NICU discharge and 
therefore cannot comment on long-term differences in ANS development between cohorts.

Because of the higher acuity at Children's National $(H I G H)$, more invasive ventilation is required, and we speculate that more handling procedures may have been required than for the relatively "well" preterm infants in the INOVA cohort (LOW). Practices such as minimal early medical handling, less invasive ventilation, early skin-to-skin contact, and prone positioning are known to impact preterm infant development and ANS maturation. ${ }^{39-41}$ Neonates with depressed autonomic function at $24 \mathrm{~h}$ of age demonstrate inappropriate responses to stimulation, impaired cerebral autoregulation during painful procedures, and vagal activation during endotracheal suctioning, potentially complicating NICU care, ${ }^{42}$ and we cannot rule out that depressed autonomic tone predisposed the Children's National cohort (HIGH) toward more medical complexity or toward the need for transfer to this referral NICU. Likewise, the cohort at INOVA (LOW) was inborn while the infants at Children's National $(H I G H)$ were all outborn. The mothers of LOW infants may have had more access and interaction with their infants than mothers of HIGH infants, who may have had to travel long distances to visit their infants in the NICU. It is not possible for us to separate the effects of these factors from the impact of the medical morbidities themselves, and likely the changes we see are multifactorial. Infants with impaired autonomic function are at increased risk for maladaptive responses during intensive care and may be more likely to have events after discharge. ${ }^{42,43}$ The LOW cohort had a significantly higher rate of cesarean-section delivery compared with the HIGH cohort. While the impact of delivery mode on HRV has not been well studied in preterm infants, our group has previously shown that delivery mode does not seem to have a significant impact on HRV metrics in full-term infants. ${ }^{15}$

Why it is that we showed significant differences in time-domain but not frequency-domain metrics of HRV between cohorts is not clear at this point, but several possible explanations warrant consideration. Time-domain metrics, including alpha 1, alpha 2, RMS1, and RMS2, may more accurately reflect ANS function under non-stationary conditions, such as critical illness. This is a question that warrants further investigation. Future, prospective studies should include real-time monitoring of preterm infants in the NICU setting and include data regarding handling procedures, skin-toskin time, time with mother's voice, and other interventions known to alter HRV, such as pacifier use. ${ }^{40,44-48}$ In summary, we found that cohorts of preterm infants with differing levels of medical complexity show differences in their ANS development, specifically for time-domain metrics of HRV. The causal nature and direction of this association is the subject of ongoing investigation.

\section{ACKNOWLEDGEMENTS}

This study was supported by the Children's National Inova Collaborative (CNICA) Research Program, through institutional support from Children's National Hospital, Washington, DC and the Inova Health System, Fairfax, VA. S.B.M. received support by Award Numbers UL1TR001876 and KL2TR001877 from the NIH National Center for Advancing Translational Sciences. Its contents are solely the responsibility of the authors and do not necessarily represent the official views of the National Center for Advancing Translational Sciences or the National Institutes of Health.

\section{AUTHOR CONTRIBUTIONS}

S.D.S. drafted the manuscript and participated in the study design, clinical data collection for the Children's National cohort, and interpretation of study results. R.B.G. participated in the study design and data analysis and provided critical review of the manuscript. S.D.B. performed statistical analysis for the study and provided critical review of the manuscript. T.A.-S. collected data at INOVA, analyzed heart rate variability data, and provided critical review of the manuscript. D.A.R. and S.I. performed clinical data collection for the Children's National cohort and provided critical review of the manuscript. L.H. was responsible for participant consent, enrollment, and clinical data collection for the INOVA cohort as well as providing critical review of the manuscript. G.L.M. and R.B. provided critical review of the manuscript. A.J.d.P. participated in the study design and critical review of the manuscript. S.B.M. designed the study, interpreted the study results, and provided critical review of the manuscript. All authors have reviewed and approved the final version of this manuscript.

\section{ADDITIONAL INFORMATION}

Competing interests: The authors declare no competing interests.

Consent: Informed consent was obtained for subjects enrolled in our prospective study at Inova Fairfax Hospital, Fairfax, VA. Subjects at Children's National Hospital were enrolled in a retrospective study with a waiver of informed consent.

Publisher's note Springer Nature remains neutral with regard to jurisdictional claims in published maps and institutional affiliations.

\section{REFERENCES}

1. Mulkey, S. B. \& du Plessis, A. J. Autonomic nervous system development and its impact on neuropsychiatric outcome. Pediatr. Res. 85, 120-126 (2019).

2. Siddiqui, S. et al. An antenatal marker of neurodevelopmental outcomes in infants with congenital heart disease. J. Perinatol. 37, 953-957 (2017).

3. Haraldsdottir, K. et al. Heart rate recovery after maximal exercise is impaired in healthy young adults born preterm. Eur. J. Appl. Physiol. 119, 857-866 (2019).

4. Thiriez, G. et al. Altered autonomic control in preterm newborns with impaired neurological outcomes. Clin. Auton. Res. 25, 233-242 (2015).

5. Mulkey, S. B. \& Plessis, A. D. The critical role of the central autonomic nervous system in fetal-neonatal transition. Semin. Pediatr. Neurol. 28, 29-37 (2018).

6. Mulkey, S. B. et al. Autonomic nervous system depression at term in neurologically normal premature infants. Early Hum. Dev. 123, 11-16 (2018).

7. Yiallourou, S. R. et al. The development of autonomic cardiovascular control is altered by preterm birth. Early Hum. Dev. 89, 145-152 (2013).

8. Patural, H. et al. Autonomic cardiac control of very preterm newborns: a prolonged dysfunction. Early Hum. Dev. 84, 681-687 (2008).

9. Mulkey, S. B. et al. Autonomic nervous system maturation in the premature extrauterine milieu. Pediatr. Res. https://doi.org/10.1038/s41390-020-0952-0 (2020).

10. Ulusar, U. D. et al. Adaptive rule based fetal QRS complex detection using Hilbert transform. Conf. Proc. IEEE Eng. Med Biol. Soc. 1, 4666-4669 (2009).

11. Kota, S. et al. Identification of QRS complex in non-stationary electrocardiogram of sick infants. Comput. Biol. Med. 87, 211-216 (2017).

12. Govindan, R. B. et al. Detrended fluctuation analysis of non-stationary cardiac beat-to-beat interval of sick infants. EPL (Europhys. Lett.) 108, 40005 (2014).

13. Govindan, R. B. Detrended fluctuation analysis using orthogonal polynomials. Phys. Rev. E 101, 010201 (2020).

14. Govindan, R. B., Preissl, H., Eswaran, H., Campbell, J. Q. \& Lowery, C. L. Detrended fluctuation analysis of short data sets: ana pplication to fetal cardiac. Phys. $D$ Nonlinear Phenom. 226, 23-31 (2007).

15. Mulkey, S. B. et al. The effect of labor and delivery mode on electrocortical and brainstem autonomic function during neonatal transition. Sci. Rep. 9, 11020 (2019).

16. Fyfe, K. L. et al. The effect of gestational age at birth on post-term maturation of heart rate variability. Sleep 38, 1635-1644 (2015).

17. Karin, J., Hirsch, M. \& Akselrod, S. An estimate of fetal autonomic state by spectral analysis of fetal heart rate fluctuations. Pediatr. Res. 34, 134-138 (1993).

18. Porges, S. W. in The Polyvagal Theory (ed. Schore, A. N.) 347 (WW Norton \& Company, 2011).

19. Longin, E. et al. Maturation of the autonomic nervous system: differences in heart rate variability in premature vs. term infants. J. Perinat. Med. 34, 303-308 (2006).

20. Mulkey, S. B. et al. Heart rate variability is depressed in the early transitional period for newborns with complex congenital heart disease. Clin. Auton. Res. 30, 165-172 (2020).

21. Clairambault, J. et al. Heart rate variability in normal sleeping full-term and preterm neonates. Early Hum. Dev. 28, 169-183 (1992).

22. Barbe, M. F. \& Levitt, P. The early commitment of fetal neurons to the limbic cortex. J. Neurosci. 11, 519-533 (1991).

23. DiPietro, J. A., Costigan, K. A. \& Voegtline, K. M. Studies in fetal behavior: revisited, renewed, and reimagined. Monogr. Soc. Res. Child Dev. 80, vii-vii94 (2015).

24. DiPietro, J. A. et al. Fetal neurobehavioral development. Child Dev. 67, 2553-2567 (1996).

25. Lucchini, M. et al. Novel heart rate parameters for the assessment of autonomic nervous system function in premature infants. Physiol. Meas. 37, 1436-1446 (2016). 
Autonomic development in preterm infants is associated with morbidity of... SD Schlatterer et al.

26. Malliani, A., Lombardi, F. \& Pagani, M. Power spectrum analysis of heart rate variability: a tool to explore neural regulatory mechanisms. Br. Heart J. 71, 1-2 (1994).

27. Valenza, G. et al. Measures of sympathetic and parasympathetic autonomic outflow from heartbeat dynamics. J. Appl. Physiol. 125, 19-39 (2018).

28. Al-Shargabi, T. et al. Changes in autonomic tone in premature infants developing necrotizing enterocolitis. Am. J. Perinatol. 35, 1079-1086 (2018).

29. Joshi, R. et al. Predicting neonatal sepsis using features of heart rate variability, respiratory characteristics, and ECG-derived estimates of infant motion. IEEE J. Biomed. Health Inf. 24, 681-692 (2020).

30. Griffin, M. P. et al. Heart rate characteristics: novel physiomarkers to predict neonatal infection and death. Pediatrics 116, 1070-1074 (2005).

31. Griffin, M. P., Lake, D. E. \& Moorman, J. R. Heart rate characteristics and laboratory tests in neonatal sepsis. Pediatrics 115, 937-941 (2005).

32. Vandenbroucke, L. et al. Chorioamnionitis following preterm premature rupture of membranes and fetal heart rate variability. PLOS ONE 12, e0184924 (2017).

33. Bohanon, F. J. et al. Heart rate variability analysis is more sensitive at identifying neonatal sepsis than conventional vital signs. Am. J. Surg. 210, 661-667 (2015).

34. Mulkey, S. B. et al. Heart rate variability is depressed in the early transitional period for newborns with complex congenital heart disease. Clin. Auton. Res. 30, 165-172 (2020).

35. Goudjil, S. et al. Patent ductus arteriosus in preterm infants is associated with cardiac autonomic alteration and predominant parasympathetic stimulation. Early Hum. Dev. 89, 631-634 (2013).

36. Metzler, M. et al. Pattern of brain injury and depressed heart rate variability in newborns with hypoxic ischemic encephalopathy. Pediatr. Res. 82, 438-443 (2017).

37. Massaro, A. N. et al. Heart rate variability in encephalopathic newborns during and after therapeutic hypothermia. J. Perinatol. 34, 836-841 (2014).
38. Reich, D. A. et al. The effect of unilateral stroke on autonomic function in the term newborn. Pediatr. Res. 85, 830-834 (2019).

39. Gomes, E. et al. Autonomic responses of premature newborns to body position and environmental noise in the neonatal intensive care unit. Rev. Bras. Ter. Intensiv. 31, 296-302 (2019).

40. Feldman, R., Rosenthal, Z. \& Eidelman, A. I. Maternal-preterm skin-to-skin contact enhances child physiologic organization and cognitive control across the first 10 years of life. Biol. Psychiatry 75, 56-64 (2014).

41. Sanders, M. R. \& Hall, S. L. Trauma-informed care in the newborn intensive care unit: promoting safety, security and connectedness. J. Perinatol. 38, 3-10 (2018).

42. Campbell, H. et al. Autonomic dysfunction in neonates with hypoxic ischemic encephalopathy undergoing therapeutic hypothermia impairs physiological responses to routine care events. J. Pediatr. 196, 38-44 (2018).

43. Nino, G. et al. Premature infants rehospitalized because of an apparent lifethreatening event had distinctive autonomic developmental trajectories. Am. J. Respir. Crit. Care Med. 194, 379-381 (2016).

44. Kommers, D. R. et al. Features of heart rate variability capture regulatory changes during kangaroo care in preterm infants. J. Pediatr. 182, 92.e1-98.e1 (2017).

45. Feldman, R. \& Eidelman, A. I. Skin-to-skin contact (Kangaroo Care) accelerates autonomic and neurobehavioural maturation in preterm infants. Dev. Med. Child Neurol. 45, 274-281 (2003).

46. Rand, K. \& Lahav, A. Maternal sounds elicit lower heart rate in preterm newborns in the first month of life. Early Hum. Dev. 90, 679-683 (2014).

47. Horne, R. S. et al. Dummy/pacifier use in preterm infants increases blood pressure and improves heart rate control. Pediatr. Res. 79, 325-332 (2016).

48. Arnon, S. et al. Maternal singing during kangaroo care led to autonomic stability in preterm infants and reduced maternal anxiety. Acta Paediatr. 103, 1039-1044 (2014). 